\title{
Evaluación de la calidad de la asistencia y el nivel de satisfacción de las pacientes asistidas en una Unidad Docente Asistencial de Mastología del Hospital de Clínicas “Dr. Manuel Quintela"
}

\author{
Assessing assistance quality and degree of patient satisfaction at a Breast \\ Teaching and Care Unit at Hospital de Clinicas"Dr. Manuel Quintela" \\ Avaliação da qualidade dos cuidados e do nível de satisfação dos \\ pacientes atendidos em uma Unidade de Ensino Assistido de Mastologia \\ do Hospital de Clínicas "Dr. Manuel Quintela"
}

Cecilia Cecilia

Profesor Adjunto Servicio de

Oncología Clínica.

Natalia Camejo

Asistente del Servicio de Oncología

Clínica.

Nora Artagaveytia

Profesor Adjunto del Departamento

Básico de Medicina.

Ana Laura Hernández

Profesor Adjunto del Departamento

de Psicologia Medica.

Lucía Delgado

Profesor Directora del Servicio de

Oncología Clínica.
Resumen: Introducción. En los últimos años ha adquirido gran relevancia evaluar la satisfacción de los usuarios con los servicios sanitarios, lo que se asocia en forma significativa con los resultados obtenidos en salud. La satisfacción de los pacientes juega un importante papel en el mantenimiento de la utilización de los servicios de salud, en la continuidad de la relación médico-paciente y en la adherencia a los tratamientos indicados. Objetivos. Conocer el grado de satisfacción manifestado por las pacientes asistidas en la Unidad Docente Asistencial de Mastología del Hospital de Clínicas e identificar los aspectos a mejorar. Material y métodos. Estudio prospectivo, aprobado por el comité de ética del Hospital de Clínicas, en el que se aplicó una encuesta anónima de satisfacción con 13 preguntas cerradas y una abierta y se recogieron datos sobre la edad y tipo de tratamiento oncológico recibido. Resultados. Se encuestaron 91 pacientes que concurrieron a la consulta en un período de dos meses. Las respuestas muestran un alto grado de satisfacción de las pacientes con la asistencia global recibida, con una puntuación media de 3,26 (IC 95\% 3.18-3.34) (rango: 1: pobre; 5: excelente), siendo este resultado independiente del grupo etario y del tipo de tratamiento oncológico recibido. Sin embargo el $38,5 \%$ de las pacientes que trabaja piensa que no pudo discutir el impacto de la enfermedad en su trabajo (media 1,82; IC 95\% 1,66-1,97) (rango 0: nada; 3: tanto como quise) y un $27 \%$ del total de las encuestadas piensa que no pudo discutir el impacto que su enfermedad tuvo en sus actividades diarias (media 2,13 IC 95\% 2,12 - 2,14) (rango: 0 : nada ; 3: si tanto como quise) ni en sus relaciones personales (27; 30 \%, media 2,0 IC 95\% 1,93-2,2) (rango: 0 : nada ; 3: si tanto como quise). Conclusiones. La puntuación global es muy satisfactoria, con dimensiones que deben mejorarse con un mayor énfasis en la comunicación sobre el impacto de la enfermedad en las actividades diarias, las relaciones personales y el trabajo. Consideramos importante implementar evaluaciones periódicas de la calidad de atención que permitan comparar los resultados y desarrollar un proceso de mejora continua.

Palabras clave: encuestas, satisfacción, cáncer de mama.

Abstract: Introduction. During the last years, assessing the degree of patient satisfaction of health services has become extremely important; and it is more often associated to the results obtained in terms of health. The degree of patient satisfaction plays a significant role in fostering the use of health care services, in developing the physician-patient relationship and in adhering to indicated treatments. Objective. To find out the degree of patient satisfaction at the Breast 
Teaching and Care Unit at Hospital de Clinicas and identify aspects that need improving. Materials and methods. prospective study, approved by the ethics committee of Hospital de Clinicas, where an anonymous satisfaction survey, with 1 open and 13 closed questions, was conducted. The survey also included data related to the age and oncological treatment received. Results. Over two months, 91 assisting patients were interviewed. Responses show a high degree of satisfaction of patients with the global care received, with a mean score of 3.26 (Cl $95 \% 3.18-$ 3.34) (range: 1: poor; 5: excellent), regardless of age group and the type of oncological treatment received. However, $38.5 \%$ of working patients believe that they were not able to discuss the impact of their disease at their jobs (mean 1.82; CC 95\% 1.66-1.97) (range: 0: not at all; 3: as much as I wanted) and $27 \%$ of patients consider that they were not able to discuss the impact of their disease on their daily activities (mean 2.13; CI $95 \% 2.12-2.14$ ) (range: 0: not at all; 3 : as much as I wanted) or their personal relations (27; $30 \%$, mean $2.0 \mathrm{Cl} 95 \%$ 1.93-2.2) (range: 0: not at all; 3: as much as I wanted). Conclusions. The global score is very satisfactory, and there are aspects to be improved, such as a better communication on the impact of the disease on daily activities, personal relations and work. We believe it is important to implement regular assessments of assistance quality that may enable to compare results and develop a continuous improvement process.

Keywords: surveys, satisfaction, breast cancer.

Resumo: Introdução Nos últimos anos, tornou-se muito importante avaliar a satisfação dos usuários com serviços de saúde, o que está significativamente associado aos resultados obtidos em saúde. A satisfação do paciente desempenha um papel importante na manutenção do uso dos serviços de saúde, na continuidade da relação médico-paciente e na adesão aos tratamentos indicados. Objetivos Conhecer o grau de satisfação expressado pelos pacientes atendidos na Unidade de Assistência de Ensino de Mastologia do Hospital de Clínicas e identificar os aspectos a serem melhorados. Material e métodos Estudo prospectivo, aprovado pelo comitê de ética do Hospital de Clínicas, no qual foi aplicada uma pesquisa de satisfação anônima com 13 perguntas fechadas e uma aberta e dados coletados sobre a idade eo tipo de tratamento oncológico recebido. Resultados Foram entrevistados 91 pacientes que participaram da consulta em um período de dois meses. As respostas mostram um alto grau de satisfação do paciente com a assistência geral recebida, com uma pontuação média de 3,26 (IC 95\% 3,183,34) (intervalo: 1: pobre; 5: excelente), sendo este resultado independente da faixa etária e o tipo de tratamento contra o câncer recebido. No entanto, 38,5\% dos pacientes que trabalham pensam que não poderiam discutir o impacto da doença em seus trabalhos (média 1,82, IC 95\%: 1,66-1,97) (intervalo 0: nenhum; tanto quanto eu queria) e $27 \%$ do total de entrevistados pensam que não poderiam discutir o impacto que sua doença teve em suas atividades diárias (média 2.13 IC 95\% 2.12 - 2.14) (intervalo: 0: nada ; 3: sim, tanto quanto eu queria) ou em seus relacionamentos pessoais (27; 30\%, média 2,0 IC 95\% 1,93-2,2) (classificação: 0: nada; 3: sim, tanto quanto eu queria). Conclusões $A$ pontuação geral é muito satisfatória, com dimensões que devem ser melhoradas com maior ênfase na comunicação sobre o impacto da doença nas atividades diárias, nas relações pessoais e no trabalho. Consideramos importante implementar avaliações periódicas da qualidade dos cuidados que nos permitem comparar os resultados e desenvolver um processo de melhoria contínua.

Palavras-chave: pesquisas, satisfação, câncer de mama.

Recibido: 01/11/2017 - Aceptado: 08/02/2018

Unidad Docente Asistencial de Mastología. Hospital de Clínicas “Dr. Manuel Quintela”. Facultad de Medicina. Universidad de la República. Montevideo - Uruguay. Correspondencia: E-mail: castillocecilia @ hotmail.com 


\section{Introducción}

Al igual que lo que se observa a nivel mundial, el cáncer de mama (CM) en Uruguay es por lejos el más frecuente en el sexo femenino y también la principal causa de muerte por cáncer. Cada año se registran en nuestro país unos 1840 casos nuevos y unas 650 mujeres mueren como consecuencia de esta enfermedad. ${ }^{(1,2)}$

La formación de centros en los que están presentes las disciplinas abocadas al diagnóstico y tratamiento de la patología nace con la necesidad de coordinar a los diferentes especialistas y mejorar el manejo del CM. El trabajo en equipo interdisciplinario permite tener una mirada integral de los aspectos biopsicosociales de las pacientes con CM y realizar las intervenciones adecuadas, optimizando los recursos y beneficiando a las pacientes. En consonancia con este enfoque y con el fin de mejorar la calidad de la asistencia de las pacientes portadoras de patología mamaria se creó la Unidad Docente Asistencial de Mastología (UDAM) en el año 2009.

En los últimos años ha adquirido gran relevancia evaluar la satisfacción de los usuarios con los servicios sanitarios ya que se asocia en forma significativa con los resultados obtenidos en salud. Se ha comprobado que la satisfacción de los pacientes juega un importante papel en el mantenimiento de la utilización de los servicios de salud, en la continuidad de la relación médico -paciente, en la adherencia a los tratamientos indicados y a las recomendaciones de los profesionales sanitarios ${ }^{(3)}$. Por otra parte, la valoración de la satisfacción de los pacientes es actualmente uno de los puntos de mayor interés en la evaluación de los servicios sanitarios, como estrategia de mejora continua de la calidad en la atención que los centros sanitarios prestan a los usuarios.

Diversos estudios publicados que evalúan la calidad de atención en cáncer gastroesófagico (4), mama ${ }^{(5)}$, colo-rectal ${ }^{(6)}$, pulmón, próstata ${ }^{(7)}$ y ginecológicos ${ }^{(8,9)}$ han encontrado que la satisfacción de los pacientes con la información proporcionada por el personal médico sobre su enfermedad y el curso del tratamiento es un factor muy importante y que influye en la satisfacción general. Asimismo, también influyen el tiempo pasado con el médico y las habilidades interpersonales del profesional, siendo otros factores claves el tiempo de espera para obtener una cita, la empatía del personal que asiste al paciente, la continuidad de la atención prestada, y la satisfacción con el personal de enfermería ${ }^{(10,11)}$.

Un estudio publicado en el 2014 ha evaluado la relación entre la satisfacción con la calidad del servicio reportada por pacientes portadoras de CM y el pronóstico de la enfermedad. Se administró para ello un cuestionario a 1521 pacientes asistidas en tres centros de cáncer de los Estados Unidos que cubrió varias dimensiones de la satisfacción de los pacientes. En este estudio, 836 pacientes habían sido diagnosticadas recientemente, y 685 habían sido tratadas previamente, incluyendo pacientes en todos los estadios de la enfermedad. Un total de 1.106 $(72,7 \%)$ pacientes se encontraban muy satisfechos con la calidad general del servicio , mientras que $415(27,3 \%)$ no lo estaban. En un análisis univariado y multivariado, las pacientes totalmente satisfechas demostraron significativamente menor mortalidad (análisis multivariado $H R=0,71$; IC del 95\% 0,57 a 0,87; $p=0,001$ ) en comparación con aquellas que no estaban completamente satisfechas, demostrándose que en este estudio la satisfacción fue un predictor independiente de sobrevida. Los autores han planteado como hipótesis que esta relación favorable entre satisfacción y sobrevida podría estar dada por: la influencia biológica de experimentar emociones positivas (que por ejemplo podrían derivar en aumento de la función inmune) así como también que la mayor satisfacción podría favorecer que los pacientes puedan realizar en forma más completa aquellos tratamientos que prolongan la vida y ello implique un mejor pronóstico. Por otra parte no fue evaluado si esta asociación favorable podría darse porque los pacientes con mejor estado de salud son lo que pueden evaluar su grado de satisfacción y en este caso la asociación entre satisfacción y sobrevida se trataría de una falsa percepción , si bien en este estudio los resultados fueron controlados por estadio y tratamientos previos recibidos ${ }^{(12)}$

Es entonces que en nuestra Unidad de Mastología nos interesó conocer la perspectiva de las pacientes en relación a los cuidados que reciben, lo que además nos permitirá implementar las modificaciones necesarias para lograr una mayor calidad de la atención en salud, compromiso, motivación y satisfacción de las usuarias así como de los diferentes profesionales que integramos el equipo. En este sentido se pretende caracterizar la posición del paciente frente a la atención sanitaria que recibe, valorando diferentes dimensiones funcionales y de bienestar global. La satisfacción percibida o las preferencias de las pacientes, son medidas útiles para evaluar, tanto la atención como proceso global, como al personal que la desarrolla, y resulta de gran utilidad para detectar puntos de mejora en un programa de gestión de calidad. Entre las diferentes metodologías que se utilizan para determinar la satisfacción de los pacientes se encuentran el desarrollo de cuestionarios o encuestas de opinión, que son apropiadas para aquellas organizaciones que prestan un servicio. Las encuestas actualmente se consideran una herramienta fundamental para conocer la opinión de las personas y es el método más 
ampliamente utilizado para la determinación objetiva y sistemática de la satisfacción de los pacientes de cáncer con la asistencia sanitaria brindada ${ }^{(10)}$. Es importante considerar los diferentes tipos de pacientes por ejemplo los pacientes que reciben diferentes modalidades de tratamiento (por ejemplo cirugía, quimioterapia, radioterapia y/u hormonoterapia) de los que reciben un único o dos tratamientos, pudiendo esto influir en los resultados. Así mismo debemos de recordar que la satisfacción es una variable acumulada, es decir, no depende de un momento puntual, sino que es la suma de múltiples contactos con la unidad. Tomando como referencia todas las consideraciones antes mencionadas, parece claro que es necesario que el profesional sanitario conozca el punto de vista, las opiniones y la calidad percibida por el paciente, con la finalidad de incrementar su satisfacción y adaptar la prestación del servicio a sus expectativas.

El objetivo de este trabajo es conocer la opinión y el grado de satisfacción manifestado por las pacientes asistidas por CM en la UDAM del Hospital de Clínicas e identificar los aspectos a mejorar.

\section{Material y métodos}

Estudio prospectivo, aprobado por el comité de ética del Hospital de Clínicas dirigido a pacientes que recibieron tratamiento por diagnóstico de CM en cualquier estadio. Se aplicó una encuesta de 13 preguntas cerradas y una abierta, en donde el paciente realizó alguna sugerencia o comentario sobre el servicio recibido (Figura 1).

\begin{tabular}{|l|l|}
\hline 1) & ¿Piensa que su equipo médico le ha ayudado a entender la enfermedad que presenta o ha presentado? \\
\hline 2) & ¿Cuánto considera que se le han explicado sus opciones de tratamiento? \\
\hline 3) & ¿Piensa que el equipo médico que la asiste le ha dedicado tiempo suficiente? \\
\hline 4) & ¿Siente que el equipo médico la ha cuidado como persona ? \\
\hline 5) & ¿Qué tan satisfecha se encuentra usted con su equipo médico? \\
\hline 6) & ¿Piensa que los médicos entienden o han entendido sus necesidades? \\
\hline 7) & ¿Siente que en las consultas tiene tiempo suficiente para conversar con su médico? \\
\hline 8) & $\begin{array}{l}\text { Conteste únicamente la siguiente pregunta si usted trabaja actualmente } \\
\text { o trabajaba al diagnóstico de la enfermedad: }\end{array}$ \\
\hline ¿Ha podido discutir con el equipo médico el impacto que ha tenido la enfermedad en su trabajo \\
\hline ¿Ha podido discutir con el equipo médico el impacto que ha tenido la enfermedad en sus actividades diarias?
\end{tabular}

En este sentido los comentarios expresados por los pacientes durante la actividad asistencial, reclamos, etc., pueden ser útiles para identificar aspectos negativos y positivos específicos del servicio que han recibido y sirven para delimitar las dimensiones de la calidad a incluir en la encuesta. De esta forma, se elimina uno de los problemas que se presentan con frecuencia en el diseño de las encuestas al incluir fundamentalmente cuestiones que interesan exclusivamente a los profesionales y no al usuario.

El diseño de la encuesta responde a una escala ordinal donde los pacientes indican su desacuerdo o acuerdo valorando entre 1 y 3 o 1 y 4 respectivamente. En las preguntas se indaga sobre : el tiempo que se le dedica al paciente durante la consulta, la explicación que se le ofrece acerca de las distintas opciones de tratamiento, el impacto de su enfermedad en su trabajo, en sus actividades diarias y en sus relaciones personales y si se le ha permitido discutir con el equipo de salud las emociones que se han presentaron en relación a su enfermedad. Por ultimo se indaga sobre la satisfacción global con el equipo médico (pregunta 13). La misma fue completada en forma anónima y voluntaria en la sala de espera de la consulta. En ningún caso la encuesta fue desarrollada en presencia del médico. Las pacientes recibieron información oral para su adecuada cumplimentación y sólo se admitió una encuesta por paciente. Se les solicitó además a las pacientes que incluyeran datos sobre la franja etaria en la que se encontraban y cuales tratamientos habían recibido por su CM (cirugía , quimioterapia, radioterapia, hormonoterapia) Una vez completada la misma, se depositaba en un buzón exterior para garantizar el anonimato. 


\section{Resultados}

Se encuestaron a 91 pacientes de sexo femenino que concurrieron a la consulta por tratamiento o control de su enfermedad en un período de dos meses (julio - agosto del 2016). Con respecto a la edad de las encuestadas, la mayoría de las pacientes (49 pacientes: $54 \%$ ) tenían entre 40 y 70 años, como se muestra en la tabla 1.

\begin{tabular}{|l|l|}
\multicolumn{1}{|c|}{ Edad } & \multicolumn{1}{c|}{ N (\%) } \\
\hline Entre 20 y 40 años & $2(2,2)$ \\
\hline Entre 20 y 40 años & $11(12 \%)$ \\
\hline Entre 50 y 60 años & $23(25,4 \%)$ \\
\hline Entre 60 y 70 años & $15(16,5 \%)$ \\
\hline Entre 70 y 80 años & $11(12 \%)$ \\
\hline Más de 80 años & $3(3,3 \%)$ \\
\hline Sin dato & $26(28,6 \%)$ \\
\hline
\end{tabular}

De las 91 pacientes, 56 se encontraban recibiendo tratamiento adyuvante , 5 en tratamiento neoayuvante, 8 en tratamiento por la enfermedad avanzada y 22 se encontraban en control clínico.

Con respecto al tratamiento recibido por CM, 84 pacientes $(92 \%)$ recibieron tratamiento quirúrgico, 82 pacientes $(90 \%$ ) recibieron tratamiento radiante, 49 pacientes $(53,8 \%)$ recibieron tratamiento con quimioterapia , $29(32 \%)$ pacientes recibieron tratamiento con hormonoterapia.

El $55 \%$ de las pacientes encuestadas (50 pacientes) recibieron 3 o más de alguno de estos tratamientos.

Las respuestas muestran un alto grado de satisfacción de las pacientes con la asistencia global recibida, con una puntuación media de 3,26 (IC 95\% 3.18-3.34) (rango: 1: pobre; 4: excelente).

En las pacientes multitratadas, que recibieron 3 o más tratamientos la puntuación media fue de 4,13 (IC 95\% 4,02-4,25).

Una importe proporción de las encuestadas (80 pacientes,90\%) se encuentra satisfecha con el equipo médico (media 3,5, IC 95\% 3,48-3.61; rango: 0: nada; 4 mucho).

La mayoría de las pacientes (77 pacientes; $85 \%$ ) piensa que se le ha ayudado a entender su enfermedad (media 3,4, IC 95\%: 3,31-3,48;rango: 0: nada; 4 mucho ) y que se le explicaron correctamente las diferentes opciones de tratamiento (74 pacientes; 84\%, media 3,23 , IC 95\% 3,17-3,31; rango: 0 : nada ; 4: mucho ).

La mayor parte está conforme con el tiempo que el médico le dedico en la consulta (76 pacientes; $80 \%$, media 3,38,IC $95 \%$ 3,29- 3,46 rango: 0 : nada ; 4: mucho ), piensa que la ha cuidado como persona (79 pacientes; 90 \%, media 3,46 , IC 95\% 3,38-3,54 rango: 0 : nada ; 4: mucho), que entendió sus necesidades (82 pacientes; 93 \%, media 2,64 ; IC 95\% 2, 57-2,71 rango: 0 : nada ; 3 : si, tanto como quiso) y que tuvo tiempo suficiente para conversar con su médico (78 pacientes, 88,5 \%, media 2,16; IC 95\% 2,05-2,28 rango: 0 : nada ; 3: si tanto como quise). Sesenta y cuatro pacientes $(72,6 \%)$ piensa que pudo discutir con el equipo medico las emociones que presento en relación con su enfermedad (media 2,56 IC 2,49-2,65 ; rango: 0 : nada ; 3 : si tanto como quise) y 76 pacientes $(86,3 \%)$ estuvieron conformes con los cuidados administrados por el equipo medico (media 3,26, IC 95\% 3,183-3,34 rango: 0 : en la absoluto ; 4 extremadamente conforme).

Sin embargo el 38,5 \% de las pacientes que trabaja (24 de 62 pacientes) piensa que no pudo discutir el impacto de la enfermedad en su trabajo (media 1,82; IC 95\% 1,66-1,97 rango: 0 : nada ; 3: tanto como quise) y un $27 \%$ del total de las encuestadas (20 pacientes) piensa que no pudo discutir el impacto que su enfermedad tuvo en sus actividades diarias (media 2,13 IC 95\% 2,12 - 2,14 ;rango: 0 : nada ; 3 : si tanto como quise) ni en sus relaciones personales (27; $30 \%$, media 2,0 IC 95\% 1,93-2,2 ) rango: 0 : nada ; 3: si tanto como quise)

Treinta y ocho pacientes $(41,7 \%)$ añadieron comentarios escritos a la encuesta siendo la mayoría de ellos positivos (33 pacientes, 86,8 \%). Diez pacientes $(10,9 \%)$ hicieron sugerencias, la mayoría estuvieron relacionadas con el tiempo de espera a la consulta, sugiriendo acortar el mismo. 


\section{Discusión}

Como fue mencionado anteriormente conocer el grado de satisfacción de las pacientes que se asisten en nuestra unidad nos permitirá mejorar la calidad de la asistencia brindada. Las pacientes que se encuentran conformes con la atención recibida desarrollan una mejor relación con su médico, tienen una mayor adherencia a los tratamientos indicados y a las recomendaciones de los profesionales, lo cual puede asociarse a un mejor pronóstico.

Los resultados de las encuestas realizadas arrojan una puntuación global satisfactoria en lo vinculado a:

a) la asistencia global recibida, aún en pacientes multitratadas lo que hace suponer la satisfacción es independiente del número de tratamientos recibido

b) la satisfacción con el equipo médico

c) la comunicación con el médico para el entendimiento de la enfermedad y de las opciones de tratamiento

d) el tiempo dedicado a la consulta

e) el cuidado del paciente como persona

f) el entendimiento de las necesidades del paciente

g) el tiempo dedicado por el médico para conversar

h) la discusión de emociones presentadas en relación a la enfermedad

i) los cuidados administrados por el médico

Por otro lado los mismos revelaron niveles de satisfacción relativamente bajos en algunos aspectos: la comunicación sobre el impacto que tuvo la enfermedad en las actividades diarias que desarrollan las pacientes, en sus relaciones personales y en su trabajo.

Esto podría tener varias explicaciones, en primer lugar, estos problemas no pueden ser abordados en profundidad durante el tiempo de la consulta debido al importante volumen de información relativa al diagnóstico y tratamiento que se brinda. También debemos tener en cuenta el incremento en el número de pacientes asistidas en los últimos dos años en nuestra Unidad lo que puede implicar menor tiempo para discutir estos aspectos.

Dado que en esta etapa se realizó una evaluación global, no se consideró el vínculo de los resultados con el estadio de la enfermedad, las comorbilidades de las pacientes, ni con la intencionalidad del tratamiento (tratamiento paliativo vs curativo). Es por ello y porque las encuestas se realizaron en forma anónima y voluntaria, que dentro de las limitaciones de nuestro trabajo, se encuentra el hecho de que no pudo establecerse la relación entre el nivel de satisfacción de las pacientes y las características clínicas de las mismas (comorbilidades, estado general, nivel socio económico y cultural), las características tumorales (extensión de la enfermedad, perfil biológico) ni con la intencionalidad y duración del tratamiento (paliativo vs curativo).

En un futuro pensamos será muy beneficioso el diseñar una serie de preguntas, que la paciente podrá responder en la sala de espera en forma relativamente rápida, con el fin de detectar más rápidamente a las pacientes que requieran una entrevista con la psicóloga de nuestra unidad con el fin de brindarle a las pacientes el espacio y el tiempo necesario para poder hablar del impacto que su enfermedad tuvo en su ámbito laboral y familiar. También las pacientes que lo requieren podrán ser derivarlas a colegas de medicina laboral con el objetivo de facilitar la reincorporación laboral y la readaptación de las pacientes, especialmente si tras la enfermedad, los procedimientos quirúrgicos empleados o las terapias aplicadas han quedado secuelas limitantes para su capacidad laboral o bien existen riesgos potenciales a valorar en su trabajo

Dado los comentarios planteados en la pregunta abierta hemos visto que otro punto a mejorar es el tiempo que esperan los pacientes para ingresar a la consulta desde que registran su llegada en el Departamento de Oncología. En ese sentido se están tratando de implementar medidas para disminuirlo como agendar pacientes con un horario de consulta, por ejemplo cuatro pacientes cada hora. Sabemos que uno de los factores que influyen en el tiempo de espera a la consulta es la demora en obtener resultados de paraclínica humoral realizada en ese día, necesaria para coordinar los tratamiento oncológicos ; en relación a esto se está implementando actualmente que independientemente del resultado pueda coordinarse igualmente el tratamiento y esto sea confirmado posteriormente al paciente vía telefónica cuando se conozcan los resultados. 
Uno de los aspectos más importante derivados del análisis de los resultados de estas encuestas de satisfacción, es que los datos obtenidos servirán para establecer una retroalimentación informativa. La valoración de las respuestas, así como las sugerencias han permitido conocer las expectativas de las usuarias. Este conocimiento se debe transformar en una oferta de servicios adecuada al mismo, es decir, en calidad asistencial. La puntuación global obtenida fue satisfactoria, aunque mejorable, siendo necesario realizar futuras encuestas que permitan comparar los resultados y evaluar lo realmente importante que es la mejora continua.

\section{Conclusiones}

La puntuación global es satisfactoria, con dimensiones que deben mejorarse con un mayor énfasis en la comunicación sobre el impacto de la enfermedad en las actividades diarias, las relaciones personales y el trabajo. La encuesta ha permitido identificar aspectos que, desde la perspectiva de las pacientes, se consideran como necesarios de abordar en mayor medida dentro del proceso de atención en la UDAM. Consideramos importante implementar evaluaciones periódicas de la calidad de atención que permitan comparar los resultados y lograr un proceso de mejora continua.

\section{Bibliografía}

1- Muñoz MJ. Impacto del cáncer de mama en Uruguay: período 2006-2010. Tendencias Med 2013; 21(43): $133-5$

2- Uruguay. Comision Honoraria de Lucha contra el Cancer. Informe Anual.Periodo 2007-2011(Fecha acceso: 6/08/2017).Disponible en: http://www.comisioncancer.org.uy/uc_394_1.html

3- Hermann, R., Ettner, S, Dorwart, R. The influence of psychiatric disorders on patients' ratings of satisfaction with health care. Medical Care. 1998; 36(5): 720-727.

4- Kavadas V, Barham CP, Finch-Jones MD, Vickers J, Sanford E, Alderson D, et al. Assessment of satisfaction with care after inpatient treatment for oesophageal and gastric cancer. Br J Surg. 2004; 91:719-723.

5- Defossez G, Mathoulin-Pelissier S, Ingrand I, Gasquet I, Sifer-Riviere L, Ingrand P. Satisfaction with care among patients with non-metastatic breast cancer: development and first steps of validation of the REPERES-60 questionnaire. BMC Cancer. 2007; 7:129.

6- Mathiesen TP, Willaing I, Freil M, Jørgensen T, Andreasen AH, Ladelund S, et al.How do patients with colorectal cancer perceive treatment and care compared with the treating health care professionals? Med Care. 2007; 45:394-400.

7- Sherlaw-Johnson C, Datta P, McCarthy M. Hospital differences in patient satisfaction with care for breast, colorectal, lung and prostate cancers. Eur J Cancer. 2008 ;44:1559-1565.

8- Vashisht A, Domoney CL, Handscomb K, Smith JR, Bridges JE. Patient satisfaction with the care provided at a gynaecology oncology clinic. J Obstet Gynaecol. 2000;20:183-184.

9- Von Gruenigen VE, Hutchins JR, Reidy AM, Gibbons HE, Daly BJ, Eldermire EM, Fusco NL. Gynecologic oncology patients' satisfaction and symptom severity during palliative chemotherapy. Health Qual Life Outcome. 2006;4:84

10- Lis CG, Rodeghier M, Gupta D. Distribution and determinants of patient satisfaction in oncology: a review of the literature. Patient Prefer Adherence. 2009; 3:287.

11- Harper JL, De Costa AM, Garrett-Mayer E, Sterba KR. Incorporating Patient Satisfaction Metrics in Assessing Multidisciplinary Breast Cancer Care Quality. South Med J. 2015 ;108(6):372.

12- Gupta D, Rodeghier M, Lis CG. Patient satisfaction with service quality as a predictor of survival outcomes in breast cancer. Support Care Cancer. 2014;22:129-134 . 